\title{
Report on the Second International Joint Conference on Rules and Reasoning
}

\author{
Christoph Benzmüller, Xavier Parent, Francesco Ricca
}

- This article reports on the Second International Joint Conference on Rules and Reasoning (RuleML+RR 2018), which was held at the University of Luxembourg September 18-21, 2018.

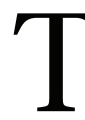

The second International Joint Conference on Rules and Reasoning (RuleML+RR 2018) ${ }^{1}$ was held September 18 to 21 at the Belval campus of the University of Luxembourg. The conference was organized as one of several collocated events of the Luxembourg Logic for AI Summit 2018 (LuxLogAI $2018)^{2}$ held September 17 to 26, 2018. LuxLogAI was substantially supported by the Luxembourg National Research Fund. ${ }^{3}$

As a novelty this year, RuleML+RR 2018 and the Fourth Global Conference on Artificial Intelligence (GCAI 2018) were collocated. A total of 224 participants attended LuxLogAI. The collaboration was thus a great success. 


\section{Promoting Al Worldwide}

AAAl sponsors many activities that promote $A$ l throughout the globe. Here are only a few of our more recent efforts:

A mailing list focusing exclusively on the discussion of US policy matters related to AI. (aaai.org/Organization/mailing-lists.php).

Support for CLAIRE - an initiative by the European Al community that seeks to strengthen European excellence in Al research and innovation.

Cooperation and affiliation with the China Computer Federation, including promotion of each other's activities, discounted membership fees, and continued exploration of new opportunities for collaboration.

Establishing regional and local chapters across the globe. (The most recent one was established in Yucatán, Mexico.)

Offering special membership rates for developing countries.

AAAI welcomes proposals for engagement of the international Al community. We look forward to receiving new opportunities and ideas for collaboration.Write to us at internationalai@aaai.org.

RuleML+RR 2018 itself provided a rich program, with five keynote talks, two tutorials, a regular program, and several subevents: an industry track, the 12th International Rule Challenge 2018, a doctoral consortium, and a poster session. A total of 33 papers were submitted, from which 10 full papers and 12 technical communications were selected. The regular papers were grouped into seven sessions on description logic, knowledge representation systems and applications, ontological reasoning, benchmarking, reasoning with modalities, complex event processing, and rule systems and applications. Presentation abstracts and a selection of slides are available online. ${ }^{4}$ Information on the various LuxLogAI conference proceedings, including the Springer Lecture Notes in Computer Science proceedings of RuleML+RR 2018, are available on the conference website. $^{5}$

The five keynote presenters at RuleML+RR 2018 were Hannah Bast (University of Freiburg), who presented Efficient and Convenient Search on Very Large Knowledge Bases; Georg Gottlob (University of Oxford), who presented Vadalog: A Language and System for Knowledge Graphs; Guido Governatori (Data61/ CSIRO), who presented Modal Rules: Extending Defeasible Logic with Modal Operators; Valeria de Paiva (Nuance Communications), who presented Bridging Trouble; and Philipp Slusallek (Saarland University/ DFKI), who presented Understanding the World with
AI: Training and Validating Autonomous Systems Using Synthetic Data.

RuleML+RR 2018 also featured two tutorials: LegalRuleML, by Guido Governatori (Data61/CSIRO) and Monica Palmirani (Bologna University), and Logic and Smart Contracts, by Bob Kowalski (Imperial College London), Miguel Calejo (logicalcontracts.com), and Fariba Sadri (Imperial College London).

The award for best paper went to Stathis Delivorias, Michel Leclere, Marie-Laure Mugnier, and Federico Ulliana for their paper On the $k$-Boundedness for Existential Rules. The Best Rule Challenge Demo award was presented to Theodoros Mitsikas, Sofia Almpani, Petros Stefaneas, Panayiotis Frangos, and Iakovos Ouranos for their presentation Formalizing Air Traffic Control Regulations in PSOA RuleML. Finally, the award for best poster went to Lukas Grätz for his contribution, Concepts as Modalities in Description Logics.

The authors of a selection of the papers with the highest reviewing scores were invited to submit an extended version of their papers to the Journal of Theory and Practice of Logic Programming for publication in a special issue on logic rules and reasoning.

As in previous years, RuleML+RR 2018 was followed by the 14th Reasoning Web Summer School, ${ }^{6}$ held September 22 to 26, 2018. This year, special emphasis was put on applications of machine learning to Web semantics.

We would like to thank our sponsors, in particular the Luxembourg National Research Fund, whose contributions allowed us to cover the costs of student participants and the invited and keynote speakers. We would also like to thank all the people who have contributed to the success of this year's special RuleML+RR 2018 and collocated events, including the organization chairs, PC members, authors, speakers, and participants.

The RuleML+RR community will again join forces with the GCAI community for a joint conference, the Bolzano Rules and Artificial Intelligence Summit (BRAIn 2019), ${ }^{7}$ to be held September 16 to 24, 2019, in Bozen-Bolzano, Italy.
1. 2018.ruleml-rr.org
2. luxlogai.uni.lu
3. RESCOM/18/12426287
4. easychair.org/smart-program/LuxLogAI2018
5. luxlogai.uni.lu/proceedings
6. 2018.ruleml-rr.org/rw.html
7. brain2019.inf.unibz.it

Christoph Benzmüller was a program cochair of RuleML+RR. He is a professor at Freie Universität Berlin, Germany.

Xavier Parent was general chair of RuleML+RR. He is a researcher at the University of Luxembourg.

Francesco Ricca was a program cochair of RuleML+RR. He is a professor at the University of Calabria, Italy. 